\title{
Effects of Different Blade Angle Distributions on Centrifugal Compressor Performance
}

\author{
Pekka Röyttä, Aki Grönman, Ahti Jaatinen, Teemu Turunen-Saaresti, and Jari Backman
}

Institute of Energy, Lappeenranta University of Technology, P. O. Box 20, 53851 Lappeenranta, Finland

Correspondence should be addressed to Pekka Röyttä, pekka.roytta@lut.fi

Received 14 April 2009; Revised 1 September 2009; Accepted 23 November 2009

Recommended by David Japikse

\begin{abstract}
A centrifugal compressor with three different shrouded 2D impellers is studied numerically. All impellers have the same dimensions, and they only differ in channel length and geometry. Noticeable differences in efficiency are observed. Two different turbulence models, Chien's $k-\varepsilon$ and $k-\omega$ SST, are compared. For this case, $k-\omega$ SST was found more realistic. The hypothesis that pressure losses in a curved duct and in an impeller passage behave similarly is suggested and found inadequate.
\end{abstract}

Copyright (C) 2009 Pekka Röyttä et al. This is an open access article distributed under the Creative Commons Attribution License, which permits unrestricted use, distribution, and reproduction in any medium, provided the original work is properly cited.

\section{Introduction}

In this paper, a hypothesis to design an impeller flow passage for a centrifugal compressor is tested. The theoretical basis of the hypothesis is presented, the equations derived, and the theory is tested using computational fluid dynamics (CFDs).

The flow in a centrifugal compressor is very complicated, with strong three-dimensional features. The subject of designing impellers is widely covered in the literature [1-5].

The blade angles of impellers are normally determined in the initial one-dimensional design phase. The blade angles are different on the impeller leading edge and on the trailing edge. Thus there has to be a transition in the blade angle along the meridional length of the blade.

In this paper, we create three different meridional blade angle distributions, keeping all of the other parameters constant. This does not necessarily lead to optimal design but illustrates the effects of the different distributions better. The performance of the resulting geometries were evaluated at three operation points presented in Table 1.

The modern practise to determine the impeller meridional blade angle distributions is to calculate the compressible inviscid flow through the impeller passage, to determine the blade loading along the blade, and then to modify the meridional blade angle distribution to produce more favourable blade loading; see for example [6].
The previous design experience gained at Lappeenranta University of Technology (LUT) tells us that the meridional blade angle distribution has a significant effect on impeller efficiency. This phenomenon has been taken into account in the inverse design approach [6-8]. However this design method has some drawbacks, as the resulting geometry is often mechanically complicated and the accuracy of the flow prediction methods available is poor. The flow is usually solved as an inviscid compressible fluid with a uniform velocity distribution at the inlet. At the moment, a complete CFD analysis of a compressor stage is computationally too expensive.

Bonaiuti et al. [9] have tried the design of experiments method to optimise transonic impeller. Part of that research involved finding the optimal meridional blade angle distribution for the inducer part of the impeller. The optimised impeller showed consistent improvement of efficiency over the whole range. The parameters were optimised separately. They found that the hub meridional blade angle distribution had a greater effect on efficiency than the shroud meridional blade angle distribution.

The use of CFD in optimization is complicated. In general the shape of the objective function is not known and function noise is always present. Thus the search algorithms have to be stochastic, although in some special applications deterministic methods have shown good results [10]. The design of a centrifugal compressor geometry includes 
TABLE 1: Mass flows used in modelling.

\begin{tabular}{lc}
\hline Low mass flow rate & $0.8 \dot{m}_{\text {des }}$ \\
Design mass flow & $\dot{m}_{\text {des }}$ \\
High mass flow rate & $1.2 \dot{m}_{\text {des }}$ \\
\hline
\end{tabular}

numerous variables, and the performance has to be evaluated at different operational points. If the design parameters $v$ are randomly varied at $n$ points the search for optimum takes $v^{n}$ evaluations. One high quality evaluation of a centrifugal compressor geometry takes hours of computational time excluding the grid generation. Thus it is necessary to severely restrict the search space and to use sophisticated algorithms to accelerate convergence [11]. In order to do search space restriction intelligently it is necessary to evaluate the influence of the different parameters to the performance of the compressor.

The secondary flows in centrifugal impellers were analysed by Brun and Kurz [12]. They have provided a model to predict secondary flows in a centrifugal compressor impeller. The conclusions of Johnston [13] are also worth reading. Equations for the secondary flows using intrinsic coordinates in turbomachinery have been developed by Horlock and Lakshminarayana $[14,15]$.

\section{Hypothesis}

The theoretical basis of this study is that an impeller passage is considered as a rotating duct with an adverse relative velocity gradient of flow. Note that the impeller in this study is shrouded. Now, the goal is to minimise the pressure loss by adjusting the meridional blade angle distribution without altering the meridional blade angle at the impeller leading or trailing edge.

We are about to model pressure loss in this rotating duct, but the correlations we use are for nonrotating ducts. This naturally is a source of some error, and we do not expect these results to be exact. We shall use CFD calculations to examine if the general trends in flows agree with our hypothesis.

The pressure loss in such a duct comprises two elements, the pressure loss due to surface friction, and the pressure loss due to the shape of the duct. To calculate the pressure loss due to skin friction we shall define the hydraulic diameter of the duct

$$
d_{h}=4 \frac{A}{P}
$$

The hydraulic diameter is used to calculate the Reynolds number in the duct.

$$
\operatorname{Re}_{d h}=\frac{\rho|\mathbf{V}| d_{h}}{\mu} .
$$

Now, given that we know the relative roughness of the impeller, we can determine the friction coefficient of the wall from the Colebrook-White equation [16],

$$
\frac{1}{\sqrt{\xi}}=-2 \lg \left[\frac{2.51}{\operatorname{Re} \sqrt{\xi}}+\frac{K_{\text {rel }}}{3.71}\right]
$$

where Re is the Reynolds number, and $K_{\text {rel }}$ is the relative surface roughness.

Now the pressure loss due to skin friction over the length of the channel $e$ can be stated as follows:

$$
\Delta p_{f l}=\int_{l} \xi \frac{\rho|\mathbf{V}|^{2}}{2} d l
$$

The integral form is essential, as the Reynolds number changes through the channel.

The pressure losses from the duct shape are due to the secondary flows induced by the geometry of the duct. The losses are higher with an increased turning angle [16]. The data by Kast [16] suggests that at low turning angles, the turning radius does not play a significant role. Thus for low turning angles the shape loss is

$$
k_{s l}=k(\Delta \beta)
$$

Further we assume that there is linear dependency between the turning angle and the losses generated,

$$
\frac{d k}{d l}=s\left|\frac{d \beta}{d l}\right|
$$

where $s$ is constant. This is implausible for wider ranges of $\Delta \beta$, but here we are interested only about one $\Delta \beta$. This is of importance because we will, once again, resort to the integral form to define the effects of shape loss,

$$
\begin{aligned}
\Delta p_{s l} & =\int_{l} \frac{d k}{d l} \frac{\rho|\mathbf{V}|^{2}}{2} d l, \\
\Longrightarrow \Delta p_{s l} & =\int_{l} s\left|\frac{d \beta}{d l}\right| \frac{\rho|\mathbf{V}|^{2}}{2} d l .
\end{aligned}
$$

This is necessary for the same reasons as in (4). It should be noted that now the total $k$ is the same for the same amount of bending regardless of the length of the bend. However, if the derivative of $\beta$ changes sign in between the $k_{s l}$ is larger for the same absolute value of angle change.

The velocity used to calculate the anticipated losses of different bends is computed assuming the lossless diffusion while fluid is considered as an ideal gas.

As the longer channel induces larger losses, one could think that the shortest possible channel is the most efficient. This, however, is not the case in centrifugal compressors. Blade backsweep has been, without a doubt, proven superior over radial vanes [17]. That is mainly due to a more radial outflow at the impeller exit, and thus, less work is done on tangential acceleration. If the blade angle turns radically at inlet, the length of the passage clearly increases. On the other hand, if the blade is turned very abruptly at the end to meet the desired backsweep, we lose the positive effects of the backsweep, as the flow will separate from the blade surface. The designer should be able to strike the balance between these two effects. 
TABle 2: Main design parameters of the studied centrifugal compressor.

\begin{tabular}{lcc}
\hline Compressor pressure ratio & - & 1.635 \\
Mass flow rate & $\mathrm{kg} / \mathrm{s}$ & 3.045 \\
Rotating speed & $\mathrm{rpm}$ & 9600 \\
$d_{2} / d_{3}$ & - & 0.616 \\
\hline
\end{tabular}

TABLE 3: Absolute lengths of impeller passages at midspan relative to the flow passage length of case 1 .

\begin{tabular}{lc}
\hline Case 1 & $l_{1}$ \\
Case 2 & $1.03 l_{1}$ \\
Case 3 & $0.97 l_{1}$ \\
\hline
\end{tabular}

\section{Methods}

Three geometries were studied with the CFD solver Finflo in order to investigate the effect of the blade angle distribution. Effects of the turbulence model and grid density were also studied. All three geometries were modelled with three mass flows.

3.1. Geometry Cases. The compressor studied in this paper is a $2 \mathrm{D}$ shrouded impeller consisting of an inlet part, 18 full blades, and a parallel wall vaneless diffuser. The main design parameters of the compressor are shown in Table 2. The studied compressor is the second stage of a two-stage industrial compressor.

Three different meridional blade angle distribution shapes, shown in Figure 1, were studied numerically. All geometries had similar inlet and outlet $\beta$-angles. The original distribution, referred to later as case 1 , was meridionally linear; whereas the second distribution (case 2) turned more closer to the blade trailing edge, and the third geometry (case 3) turned more closer to the blade leading edge. The absolute lengths of flow passages are presented in Table 3. Surface grid of the impeller in case 1 without a shroud is shown in Figure 2, and every other gridline is visible for the sake of clarity. All three grids consisted of three calculation blocks, which were inlet part, blade channel, and diffuser. Every grid had the same amount of cells and similar node distribution, which made them comparable.

3.2. Numerical Methods. Finflo is a multigrid Navier-Stokes solver employing the finite-volume method for spatial discretization. This study employs Roe's flux-difference splitting method [18] for inviscid fluxes. Convective fluxes are discretized by a second-order upwind scheme, and also a flux limiter is applied for the studied problem.

Turbulence is modelled with the $k-\omega$-SST model [19] in all cases and with Chien's $k-\epsilon$ model [20] in two cases and in the grid dependency study. Wall functions are not used.

The total enthalpy and momentum distributions are used as inlet boundary conditions, and the static pressure is extrapolated from the computational domain. Inlet flow conditions are from the one-dimensional design of the studied compressor. The static pressure is used as an outlet

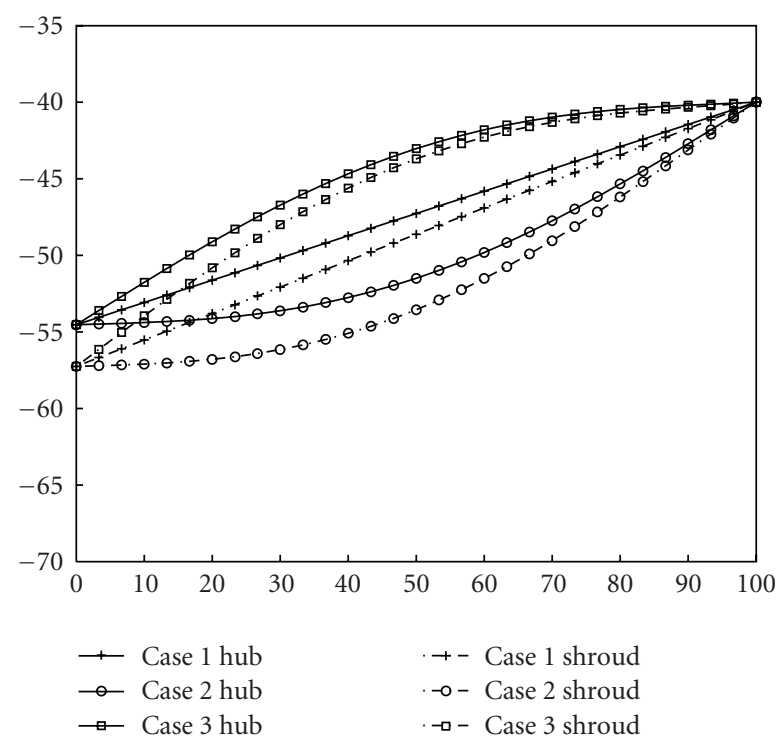

Figure 1: Meridional blade angle distributions of the studied compressors.

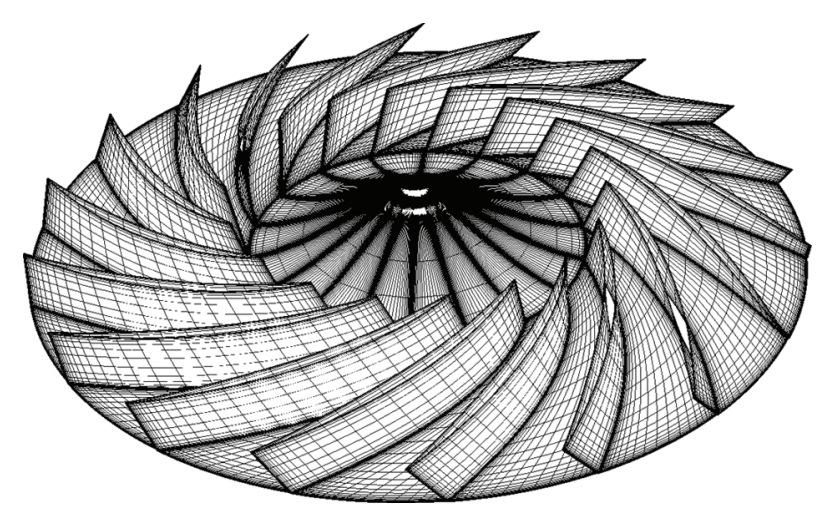

FIGURE 2: Surface grid of the impeller in case 1 without a shroud. Every other gridline is visible for the sake of clarity. The impeller is presented as a whole, but the calculations were done using one flow passage.

boundary condition at the diffuser outlet. The mass flow difference between the inlet and outlet domains and the maximum change in density are used as convergence criteria.

3.3. Grid Dependency. In order to evaluate the grid dependency of the studied geometry, three grid densities were compared: grid 1 had 68608 cells, grid 2 had 548864 cells and grid 3 had 932736 cells. The nondimensional wall distance $y$ ${ }^{+}$was less than unity in most of the blade surfaces for the two largest grids. The maximum value of 6.2 was detected at the trailing edge of grid 3. The maximum value for $y^{+}$for the grid 1 at the blade surface was 7.4 on the trailing edge. The contours of $y^{+}$for case 1 and a plot of $y^{+}$at the blade pressure surface (white dots) at the design mass flow rate with the $k-\omega$-SST turbulence model are shown in Figure 3. Higher (more than unity) values of $y^{+}$are seen at the leading and trailing edges in relatively small areas. In overall the 


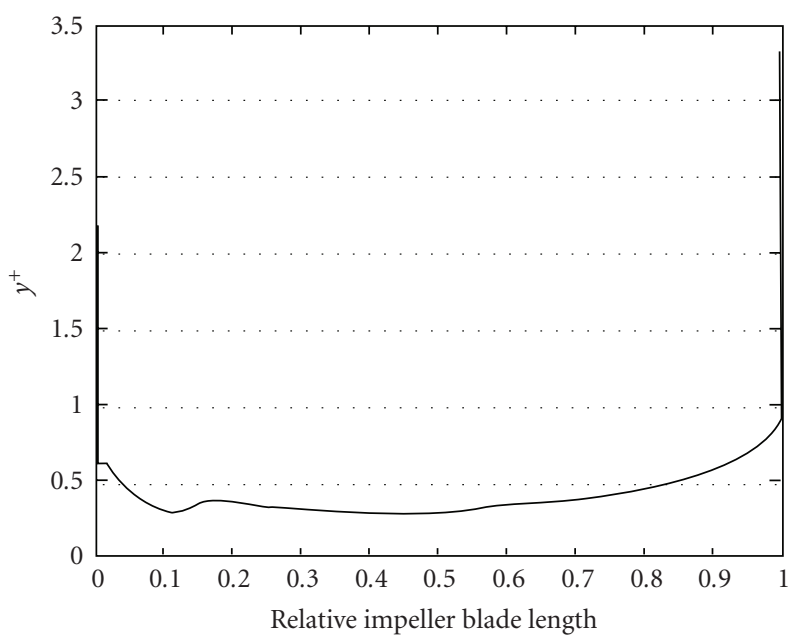

(a)

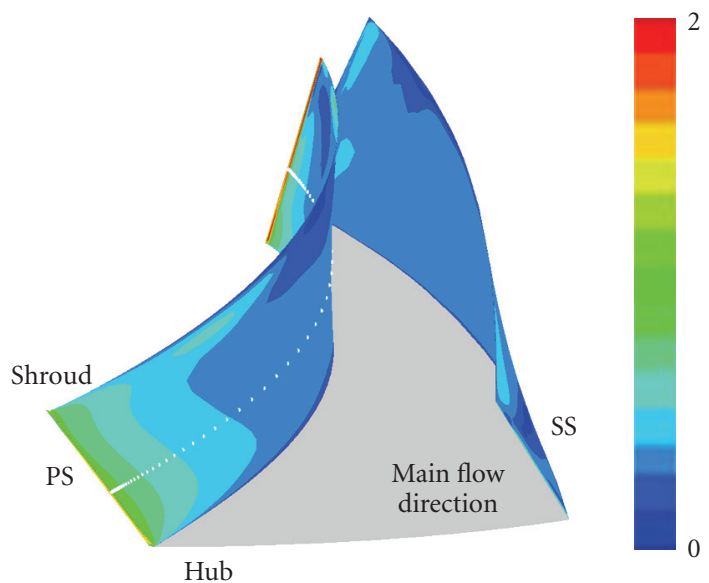

(b)

Figure 3: Contours of nondimensional wall distance $y^{+}$for case 1 at the design mass flow rate with the $k$ - $\omega$-SST turbulence model. Values at the pressure side (PS) are plotted from the leading edge to the trailing edge along the blade surface (highlighted white spots).

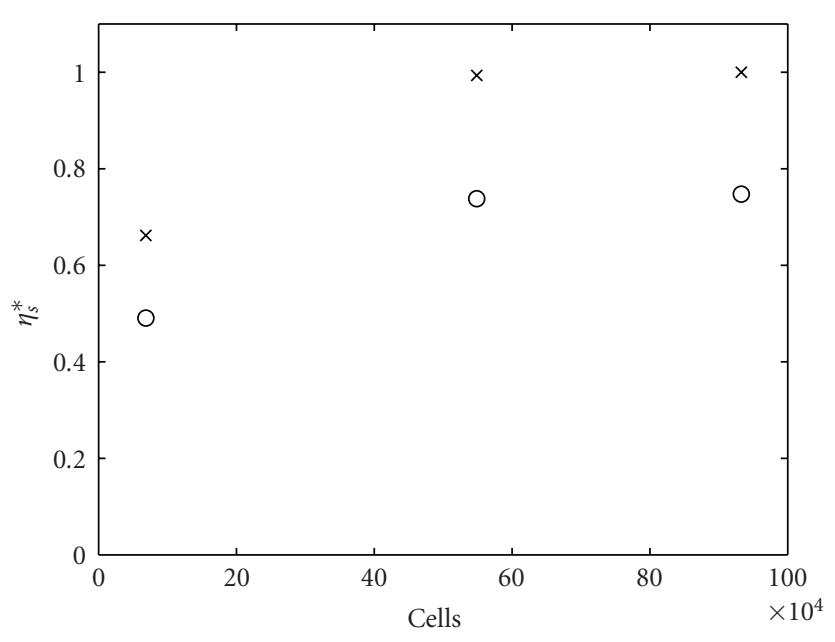

$\bigcirc \eta_{s 2, t-s}$

$\times \eta_{s 3, t-s}$

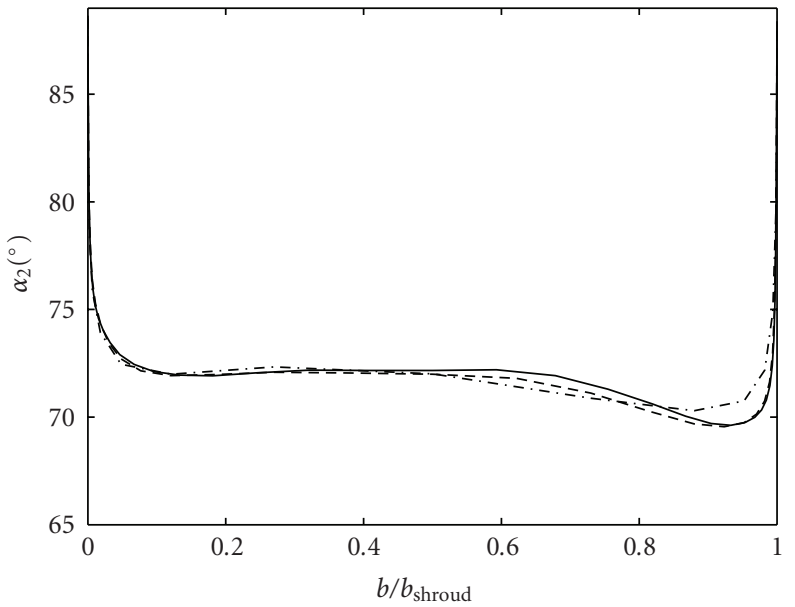

-.-- Grid 1

- - - Grid 2

- Grid 3

(a)

(b)

Figure 4: Isentropic efficiency versus grid cell number (a) and flow angle at impeller outlet with different cell counts (b). Hub is at 0 and shroud at 1 .

values are higher at the pressure side (PS) than those at the suction side (SS). Over unity values at the trailing edge might have effect on the predicted wake and loss development. The grid dependency was studied by looking at the flow angles at the impeller outlet and the total to static efficiency of the impeller and impeller-diffuser, $\eta_{s 2, t-s}$ and $\eta_{s 3, t-s}$ respectively. The comparisons were done at the design mass flow.

In the calculations, the specific isobaric heat capacity $c_{p}$ was assumed constant. Thus the efficiency for the impeller outlet can be defined as follows:

$$
\eta_{s, t-s}=\frac{T_{2 s}-T_{t 1}}{T_{2}-T_{t 1}}
$$

The static temperature after compression is calculated from the ideal gas equation

$$
T_{2 s}=T_{t 1}\left(\frac{p_{2}}{p_{t 1}}\right)^{R / c_{p}} .
$$

The efficiency and temperature at the diffuser outlet are defined correspondingly.

Based on Figure 4(a), it seems that the number of cells added from grid 2 to grid 3 does not significantly affect the estimated efficiency. To be sure, let us consider the flow angles at the impeller exit. From Figure 4(b) it is apparent that the flow angles in grids 2 and 3 are almost similar. 
TABLE 4: Theoretically calculated surface friction losses and shape losses in the impeller passages with different mass flows scaled to the design mass flow values of case 1 .

\begin{tabular}{lcc}
\hline $0.8 \dot{m}_{\text {des }}$ & Surface friction & Shape loss \\
\hline Case 1 & 0.71 & 0.64 \\
Case 2 & 0.89 & 0.62 \\
Case 3 & 0.68 & 0.68 \\
\hline$\dot{m}_{\text {des }}$ & Surface friction & Shape loss \\
\hline Case 1 & 1 & 1 \\
Case 2 & 1.25 & 0.97 \\
Case 3 & 0.94 & 1.06 \\
\hline $1.2 \dot{m}_{\text {des }}$ & Surface friction & Shape loss \\
\hline Case 1 & 1.32 & 1.44 \\
Case 2 & 1.60 & 1.39 \\
Case 3 & 1.32 & 1.53 \\
\hline
\end{tabular}

From this, it can be interpreted that grid 3 with 932736 cells is sufficient; that is, higher cell counts probably would not improve the results.

3.4. Assessment Criterion. In addition to the efficiency presented before, we use the pressure loss coefficient and pressure rise coefficient to measure the performance of the diffuser. The total pressure loss coefficient $K_{p}$ is

$$
K_{p}=\frac{p_{t 2}-p_{t 3}}{p_{t 2}-p_{2}}
$$

The total pressure loss coefficient gives information about the quality of the diffuser flow.

The static pressure rise coefficient of the diffuser $C_{\mathrm{pr}}$ is

$$
C_{\mathrm{pr}}=\frac{p_{3}-p_{2}}{p_{t 2}-p_{2}}
$$

The pressure rise coefficient tells how much of the dynamic head at the impeller outlet was recovered as static pressure rise in the diffuser.

\section{Results}

4.1. Theoretical Results. Theoretical solutions for speed distribution in Figure 5 show that case 2 has the highest average velocity and case 3 the lowest. As the flow passage is also the longest in case 2, the surface friction losses are the greatest; the relative values are presented in Table 4 . The surface friction losses in Table 4 are calculated with (4) and the shape losses with (7). The only counter intuitive result is that case 3 seems to lose its benefit of lower surface friction at off-design points. This would indicate that case 3 has lower total-to-total efficiency over the impeller, $\eta_{t-t, 2}$, than case 1 especially at off-design points.

From Figure 5 it can be interpreted that the turning the flow channel after diffusion seems to be quite a lot more efficient than before when considering shape losses according to the hypothesis.

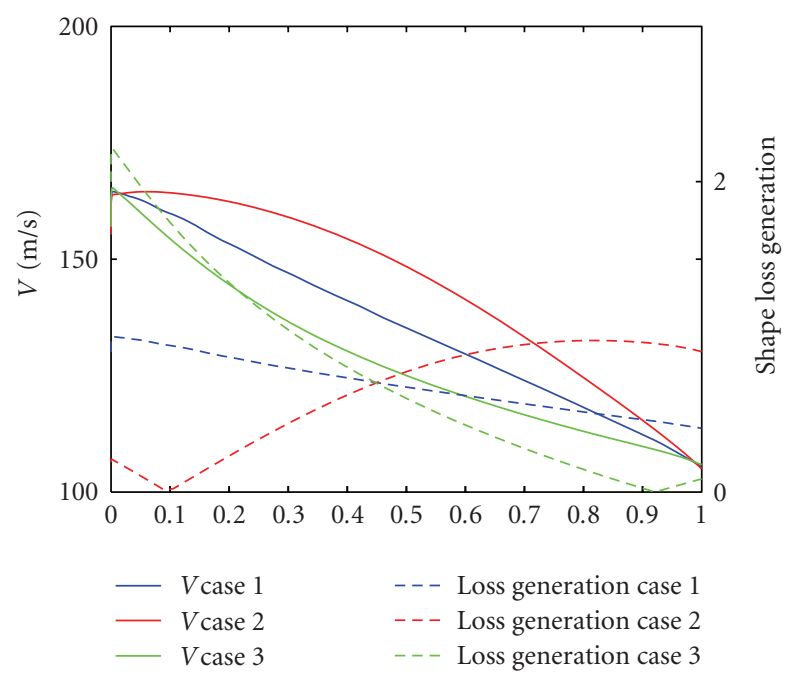

FIgure 5: The theoretical velocity distribution at design mass flow and the calculated shape loss generation.

4.2. Compressor Performance. The total to static performances of the studied cases are presented in Figure 6(a). The first case seems to have the best impeller efficiency at the design point, and consistently good efficiency at other points. Case 2 has the best performance at the low mass flow and case 3 at the high mass flow. The efficiencies are scaled to the maximum value of efficiency.

At the low mass flow rate, the stage efficiency differences are larger than the impeller efficiency differences. Although case 1 has the best impeller efficiency at the design point, case 3 has practically the same stage efficiency at the same point.

At the high mass flow rate, the impeller efficiencies of cases 1 and 3 are practically equal, but the stage efficiency of case 3 is slightly higher.

Impeller total-to-total efficiencies are presented in Figure 6(b). The efficiency of case 1 is the highest at the low and design mass flows. Case 2 has the lowest efficiency at the high and design mass flows, but is practically equal to the highest efficiency at the low mass flow.

4.3. Losses in the Impeller. In Table 5, the work done in the impeller passage at the design mass flow is presented. All values are scaled to the amount of work done in Case 1. The losses are calculated and the percentage of losses caused by the turbulent dissipation and the viscous dissipation at the wall is presented. The loss distributions between cases are very similar. The work done is calculated between the total states. Therefore no heat is conducted through the walls, it is really the amount of work done to the fluid by the impeller.

4.4. Velocity Profiles. In Figure 7, the relative velocity profiles in the flow passages are presented under the design mass flow conditions. Case 2 differs in the shape of the curve as the flow starts to accelerate early in the passage. The average relative velocity is the highest in case 2 and the lowest in case 3 . 


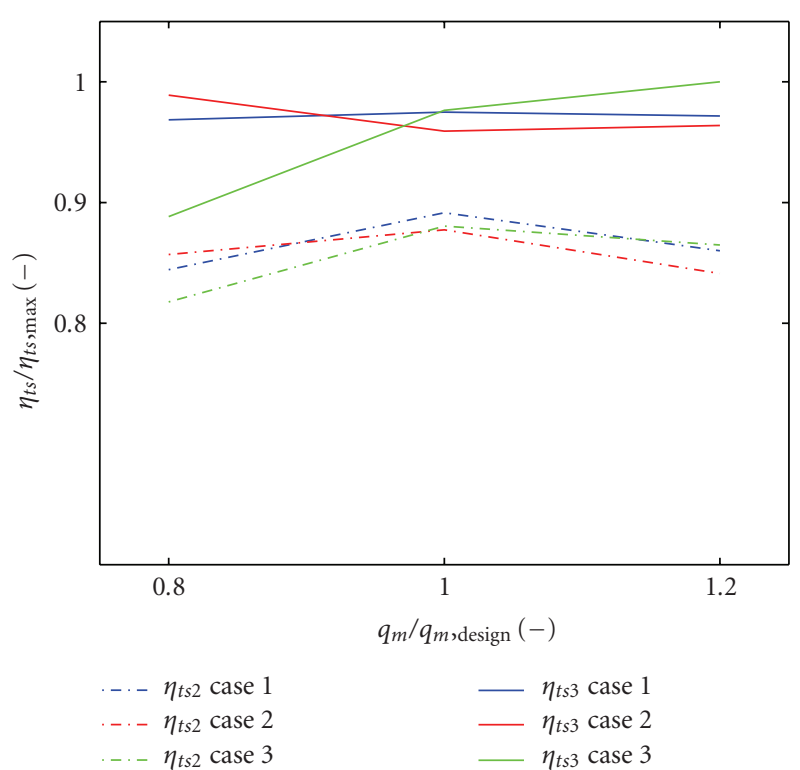

(a)

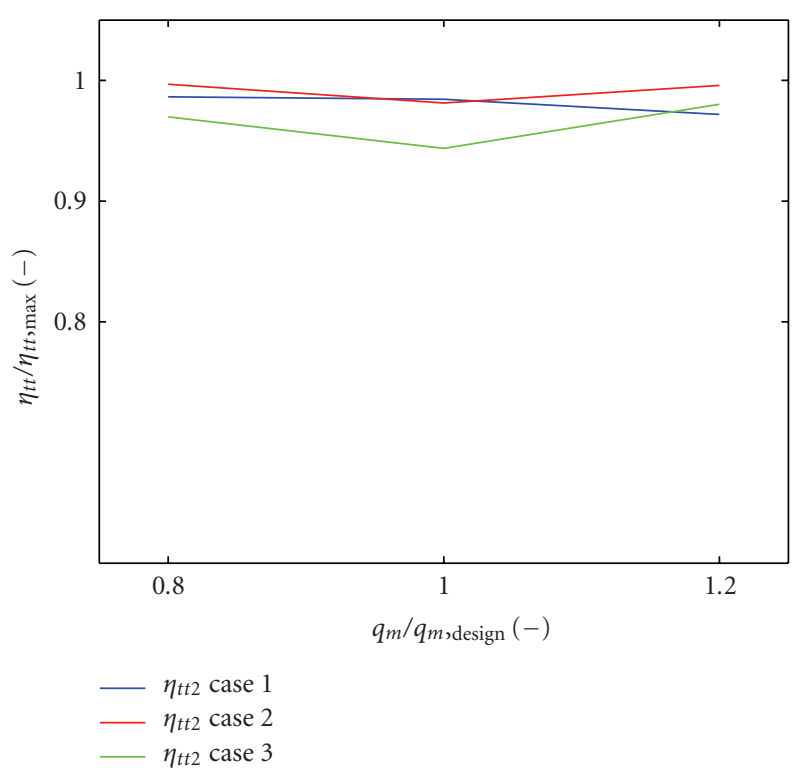

(b)

Figure 6: Isentropic total to static efficiencies (a) and isentropic total to total efficiencies over impeller (b). The efficiencies are presented relative to the maximum value.

TABLE 5: Losses in the impeller passage.

\begin{tabular}{lccc}
\hline & Case 1 & Case 2 & Case 3 \\
\hline$W_{2}$ & 1.000 & 0.983 & 1.004 \\
$W_{2 s}$ & 0.932 & 0.901 & 0.935 \\
$W_{\text {losses }}$ & 0.072 & 0.087 & 0.074 \\
\hline Losses & & & \\
Viscous & $15.8 \%$ & $15.8 \%$ & $13.4 \%$ \\
Turbulence & $21.4 \%$ & $21.8 \%$ & $19.6 \%$ \\
\hline
\end{tabular}

Also the velocities modelled with the $k-\epsilon$ turbulence model for case 1 and 2 are presented in Figure 7. They are higher than the ones modelled with $k$ - $\omega$, and the curve shape is also different.

All impellers have acceleration in the mass flow averaged velocity near the trailing edge even though the crosssectional area increases. In the flow field Figures 8(a), 8(b), and 8 (c) we see a large area of low energy wake flow close to the trailing edge at the suction side of the impeller, as can be expected. The contours of relative velocity also confirm the higher averaged velocities of case 2 over cases 1 and 3 .

In Figure 9, the mass averaged flow angle of all cases along the passage is presented alongside the actual blade angles at the hub and shroud. The flow is turned the most in case 3 and the least at case 2 . These results are derived at the design point.

When studying Figures 7 and 9 it seems that the actual flow angle changes starts to deviate quickly from the blade angle after the area starts to grow more rapidly. This seems to be due to flow separation, based on Figure 8 .

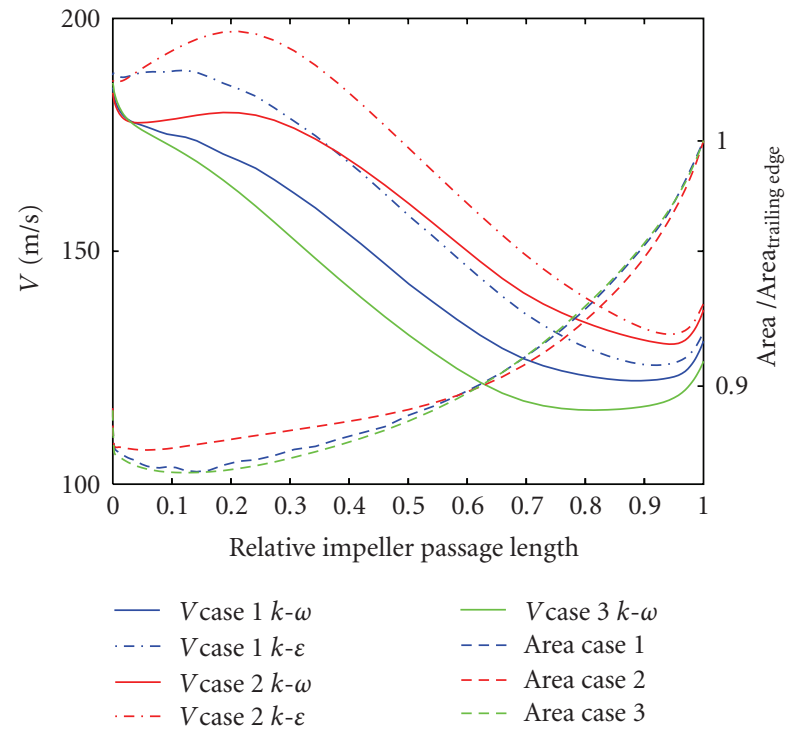

FIGURE 7: Mass flow averaged flow velocity relative to the impeller at the flow passage and the development of the cross-sectional area of the flow passage.The solid line represents the velocity distribution calculated with $k-\omega$ turbulence model. The results are obtained at the design point.

The flow field shows rather normal behaviour for a centrifugal compressor. The high energy jet flow is near the pressure side, and the low energy wake is on the suction side. Similar results can be seen in [21].

In Figure 8 cases 1 and 3 show clear low energy flow near the leading edge, unlike case 2 . This is due to flow separation. However, case 2 shows the highest mass averaged velocity in Figure 7. 


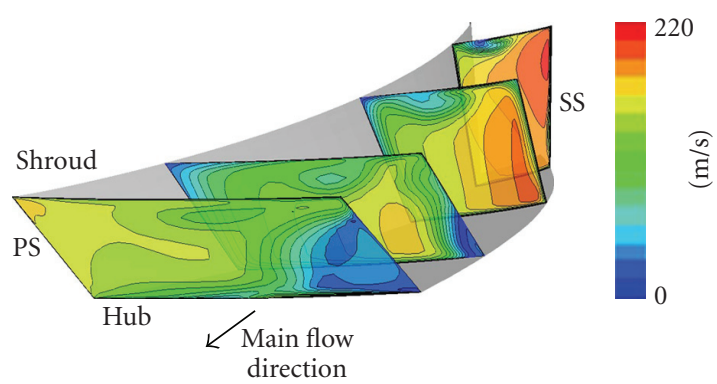

(a)

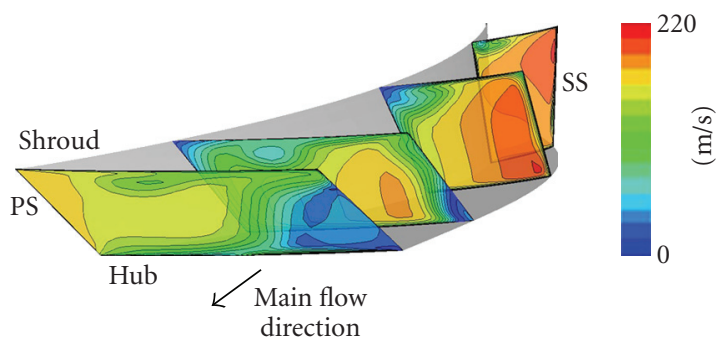

(b)

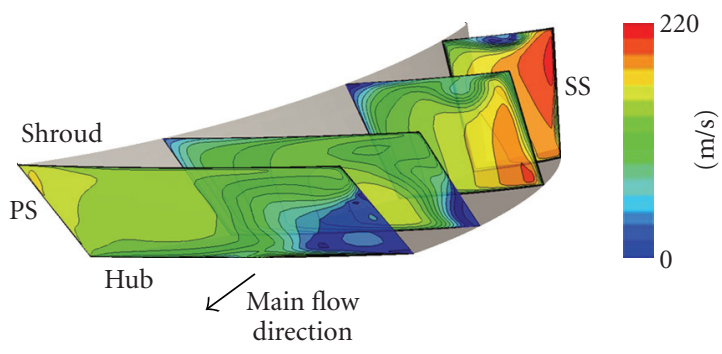

(c)

FIGURE 8: Contours of relative velocity for (a) case 1, (b) case 2, and (c) case 3 ( $k$ - $\omega$ turbulence model).

TABLE 6: Diffuser performance.

\begin{tabular}{lcccccc}
\hline & \multicolumn{2}{c}{ Case 1 } & \multicolumn{2}{c}{ Case 2 } & \multicolumn{2}{c}{ Case 3 } \\
\hline & $C_{\mathrm{pr}}$ & $K_{p}$ & $C_{\mathrm{pr}}$ & $K_{p}$ & $C_{\mathrm{pr}}$ & $K_{p}$ \\
\hline Low mass flow & 0.180 & 0.452 & 0.196 & 0.417 & 0.100 & 0.510 \\
Design mass flow & 0.125 & 0.415 & 0.125 & 0.403 & 0.145 & 0.366 \\
High mass flow & 0.173 & 0.320 & 0.198 & 0.292 & 0.208 & 0.268 \\
\hline
\end{tabular}

4.5. Blade Loading. In Figure 10, the blade loading of case 2 near the leading edge is considerably lower than in other cases and is the highest at the trailing edge. The opposite is true for case 3 . Case 1 is the most consistent, but shows small wobbling near the leading edge.

4.6. Diffuser Performance. The pressure rise coefficient of the diffuser of Case 3 is the lowest at the low mass flow rate, but the highest at other points. Cases 1 and 2 have their lowest pressure rise coefficients at the design mass flow as can be seen in Table 6.

The overall total pressure loss coefficient is higher at lower mass flow rates.

\section{Discussion}

The stage efficiency and impeller efficiency are not linearly dependent as seen in Figures 6(a) and 6(b). The theoretical results suggest that the total-to-total efficiency over the impeller of case 3 would deteriorate when compared to the case 1; the opposite is true according to Figure 6(b). Clearly, the initial hypothesis was incorrect according to CFD. The impeller efficiency does not act the way the hypothesis suggests, neither the stage efficiency. This can be seen by comparing Figure 6(b) with Table 4. This is most likely explained by the fact that the most important secondary flow phenomena responsible of the losses are due to the centrifugal force. Centrifugal force in pipe bends acts towards concave wall and in a rotating centrifugal compressor impeller it is towards the convex wall, this seems to be the significant difference why the proposed analogy fails. More importantly, superior impeller efficiency does not predict superior stage efficiency with the same diffuser, further emphasising the need to understand impeller-diffuser interaction better.

In the steady-state calculation, the continuity equation states that the mass flow through every cross-section of the impeller passage is the same. Thus, the higher mass averaged velocity can only be explained by a smaller effective flow area or smaller density. Here we operate in subsonic velocities. Thus, shock waves do not occur and the changes in temperature and pressure are rather similar for all of the wheels. Because of this, we deduce that somehow the effective flow area is reduced. This is probably due to flow separation. As seen in Figure 9, the flow breaks away from the blade direction just after the halfway mark through the channel.

Quite logically, the average speed in the flow channel is the highest when the impeller passage is the longest, as in case 2 , and the lowest when the impeller passage is the shortest, as in case 3 . This is because of the different cross-sectional areas. As the diameters of the wheels are the same and the thickness of the wanes is constant the longest passage will have the smallest average cross-sectional area.

The initial hypothesis suggested that case 2 should have been the most efficient, given that the shape losses are considerably higher than the friction losses. However, it seems to fail at the very beginning of the flow passage. Nonetheless, no clear flow separation is present near the leading edge; see Figure 8. However, later in the channel the diffusion continues the furthest but does not make up for the losses near leading edge Figure 7.

Near the leading edge, case 3 shows superior diffusion, but looses the most pressure near the trailing edge because of flow acceleration. This occurs regardless that the crosssectional area increases. The effective cross-sectional area has to decrease because of flow separation. Indeed, we see larger flow separation at the trailing edge of case 3. Without this separation, case 3 would exhibit much better performance.

Velocity profiles suggest that in case 3 , the effective crosssectional area decreases after a meridional length of 0.7 . It also makes sense that the average velocity of case 3 is the highest. 


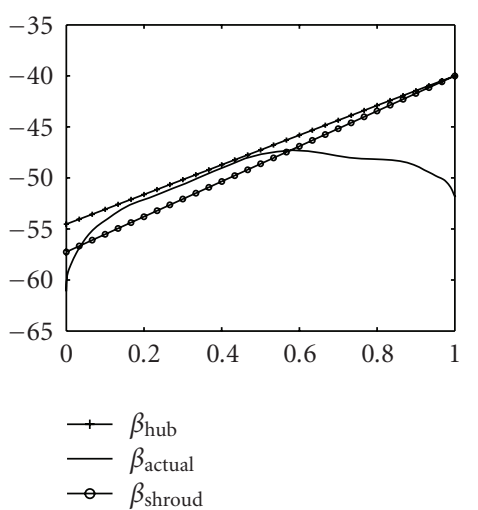

(a)
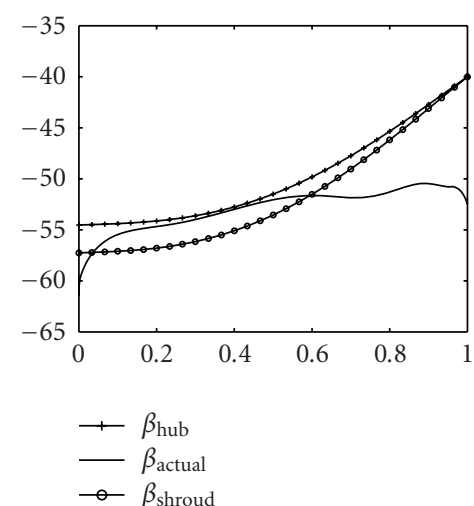

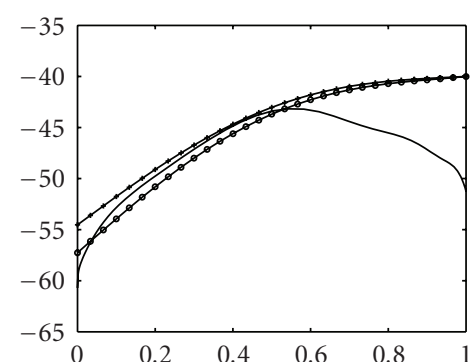

$+\beta_{\text {hub }}$
$-\beta_{\text {actual }}$
$\rightarrow-\beta_{\text {shroud }}$

(c)

Figure 9: The actual mass averaged flow angles in the impeller versus the blade angles at hub and shroud: (a) case 1, (b) case 2, and (c) case 3.

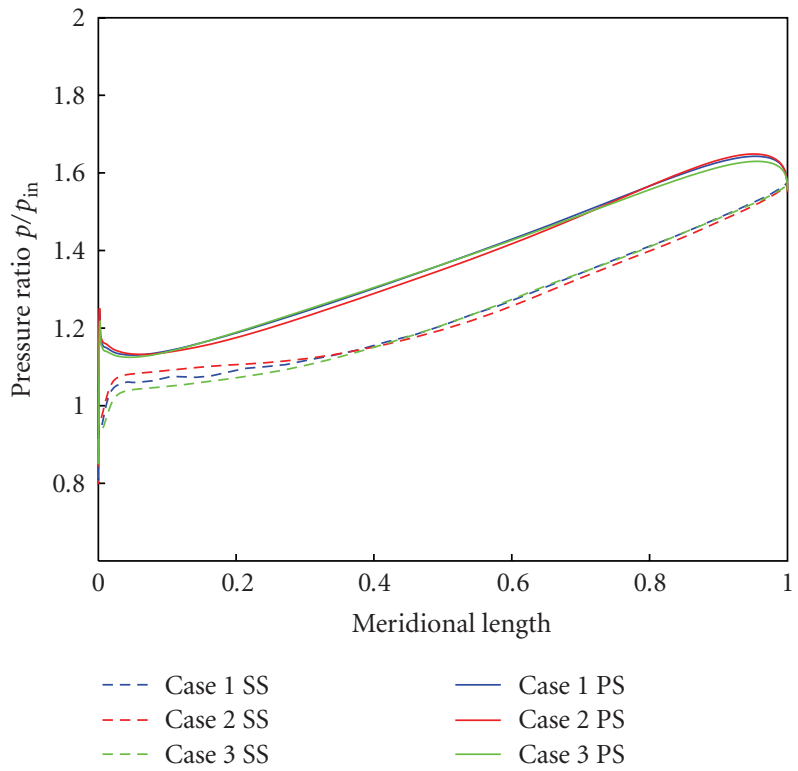

FIGURE 10: The ratio of the static pressure to the pressure at the inlet at the midspan of the flow channel on both the suction and pressure sides at design mass flow.

The diffuser pressure rise is much lower with $k-\omega$ turbulence model. This is due to earlier and more severe flow separation. It is generally known that the $k-\epsilon$-turbulence model fails to predict flow separation at the areas of the adverse pressure gradient. The difference in overall total-tostatic efficiency between the models is $14 \%$.

\section{Nomenclature}

\section{Latin alphabet}
A: Area $\left(\mathrm{m}^{2}\right)$
d: Diameter (m)
$l$ : Absolute length of flow passage $(\mathrm{m})$
$k$ : Loss coefficient

$K_{\mathrm{p}}$ : Pressure loss coefficient

$K_{\text {rel }}:$ Relative surface roughness

$K_{p}$ : Static pressure rise coefficient

$P: \quad$ Circumference $(\mathrm{m})$

$p: \quad$ Pressure $(\mathrm{Pa})$

$k: \quad$ Loss coefficient

$T: \quad$ Temperature $(\mathrm{K})$

$V:$ Relative flow velocity $(\mathrm{m} / \mathrm{s})$.

\section{Dimensionless numbers}

Re: Reynolds number.

\section{Greek alphabet}

$\eta$ : Efficiency

$\mu$ : Dynamic viscosity $(\mathrm{Pa} \cdot \mathrm{s})$

$\rho$ : Density $\left(\mathrm{kg} / \mathrm{m}^{3}\right)$

$\xi$ : Friction coefficient.

\section{Subscripts}

$\begin{array}{ll}d h: & \text { Hydraulic diameter } \\ f l: & \text { Friction loss } \\ h: & \text { Hydraulic } \\ s: & \text { Isentropic } \\ s l: & \text { Shape loss } \\ 1: & \text { Compressor inlet } \\ 2: & \text { Impeller outlet } \\ 3: & \text { Diffuser outlet } \\ t: & \text { Total } \\ t-s: & \text { Total to static } \\ t-t: & \text { Total to total. }\end{array}$

\section{Acknowledgments}

This study was done in collaboration with Ecopump Ltd. within the framework of the VIRKOOT project, and was partly funded by TEKES - the Finnish Funding Agency 
for Technology and Innovation. Part of the computational resources was provided by CSC-the IT Center for Science.

\section{References}

[1] O. E. Balje, Turbomachines: A Guide to Design, Selection and Theory, John Wiley \& Sons, New York, NY, USA, 1981.

[2] P. M. Came and C. J. Robinson, "Centrifugal compressor design," Journal of Mechanical Engineering Science, vol. 213, no. 2, pp. 139-155, 1998.

[3] S. L. Dixon, Fluid Mechanics, Thermodynamics of Turbomachinery, Pergamon Press, Oxford, UK, 3rd edition, 1989.

[4] D. Japikse, Centrifugal Compressor Design and Performance, Concepts ETI, Wilder, Vt, USA, 1996.

[5] H. I. H. Saravanamuttoo, G. F. C. Rogers, and H. Cohen, Gas Turbine Theory, Prentice-Hall, Upper Saddle River, NJ, USA, 5th edition, 2001.

[6] M. Zangeneh, M. Schleer, F. Pløger, et al., "Investigation of an inversely designed centrifugal compressor stage-part I: design and numerical verification," Journal of Turbomachinery, vol. 126, no. 1, pp. 73-81, 2004.

[7] M. Zangeneh, "A compressible three-dimensional design method for radial and mixed flow turbomachinery blades," International Journal for Numerical Methods in Fluids, vol. 13, no. 5, pp. 599-624, 1991.

[8] M. Zangeneh, "Inverse design of centrifugal compressor vaned diffusers in inlet shear flows," Journal of Turbomachinery, vol. 118, p. 385, 1996.

[9] D. Bonaiuti, A. Arnone, M. Ermini, and L. Baldassarre, "Analysis and optimization of transonic centrifugal compressor impellers using the design of experiments technique," Journal of Turbomachinery, vol. 128, no. 4, pp. 786-797, 2006.

[10] K. C. Giannakoglou and D. I. Papadimitriou, "Adjoint methods for shape optimization," in Optimization and Computational Fluid Dynamics, pp. 79-109, Springer, Berlin, Germany, 2008.

[11] R. A. Van den Braembussche, "Numerical optimization for advanced turbomachinery design," in Optimization and Computational Fluid Dynamics, pp. 147-189, Springer, Berlin, Germany, 2008.

[12] K. Brun and R. Kurz, "Analysis of secondary flows in centrifugal impellers," International Journal of Rotating Machinery, vol. 2005, no. 1, pp. 45-52, 2005.

[13] J. P. Johnston, "Effects of system rotation on turbulence structure: a review relevant to turbomachinery flows," International Journal of Rotating Machinery, vol. 4, no. 2, pp. 97-112, 1998.

[14] J. H. Horlock and B. Lakshminarayana, "Secondary flows: theory, experiment, and application in turbomachinery aerodynamics," Annual Reviews in Fluid Mechanics, vol. 5, no. 1, pp. 247-280, 1973.

[15] B. Lakshminarayana and J. H. Horlock, "Generalized expressions for secondary vorticity using intrinsic co-ordinates," Journal of Fluid Mechanics, vol. 59, no. 1, pp. 97-115, 1973.

[16] W. Kast, "Druckverlust," in VDI-Wäarmeatlas: Berechnungsbläatter fäur den Wäarmeäubergang, pp. La1-Lc9, Verein Deutscher Ingenieure, Däusseldorf, Germany, 5th edition, 1988.

[17] R. K. Turton, Principles of Turbomachinery, E. \& F. N. Spon, 1984.

[18] P. L. Roe, "Approximate Riemann solvers, parameter vectors, and difference schemes," Journal of Computational Physics, vol. 43 , no. 2 , pp. 357-372, 1981.
[19] F. R. Menter, "Zonal two-equation $k-\omega$ turbulence models for aerodynamic flows," AIAA Paper 93-2906, 1993.

[20] K. Y. Chien, "Predictions of channel and boundary-layer flows with a low-reynolds-number turbulence model," AIAA Journal, vol. 20, no. 1, pp. 33-38, 1982.

[21] F. Iancu, J. Trevino, and S. Sommer, "Numerical analysis of blade geometry generation techniques for centrifugal compressors," International Journal of Rotating Machinery, vol. 2007, Article ID 48683, 7 pages, 2007. 

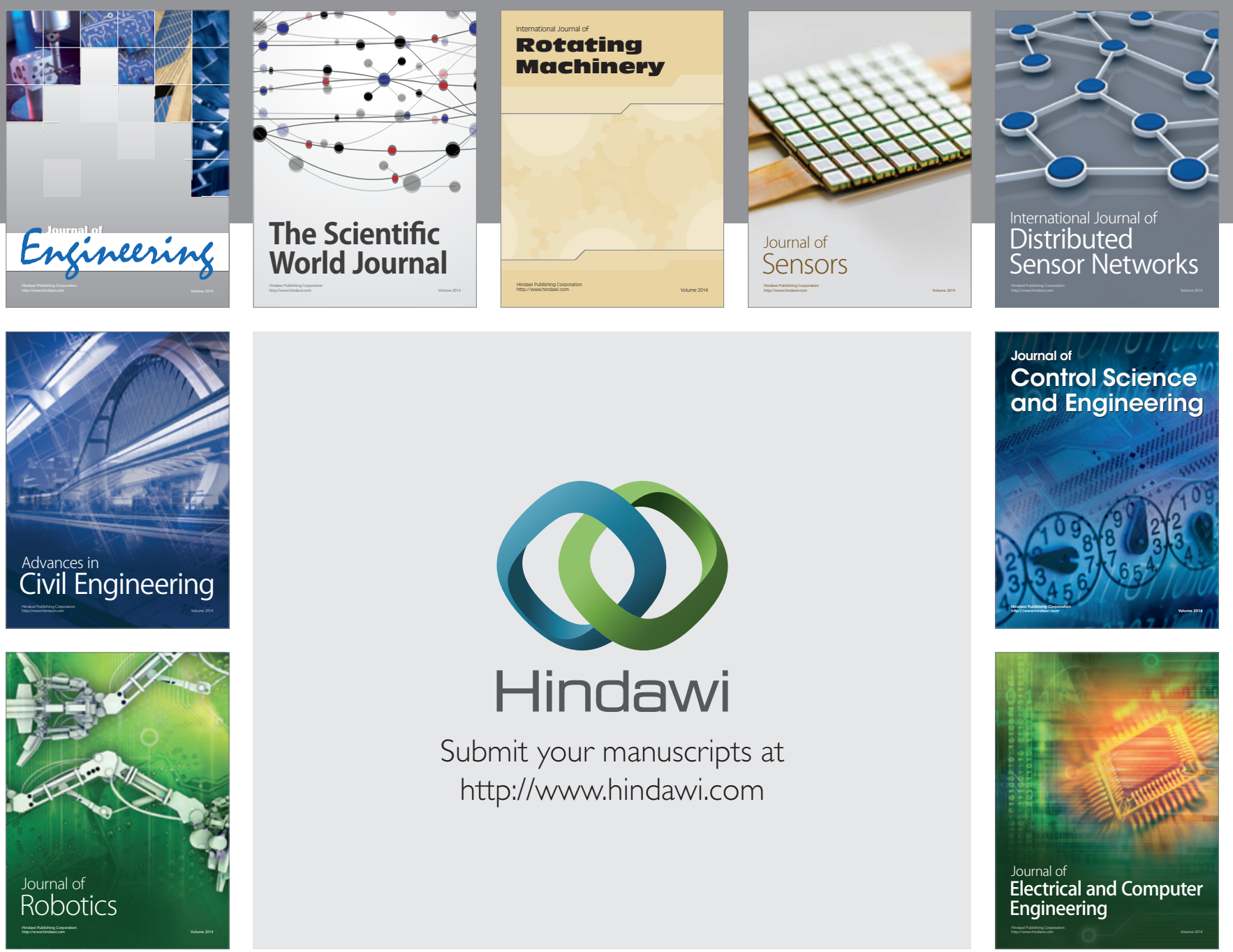

Submit your manuscripts at

http://www.hindawi.com
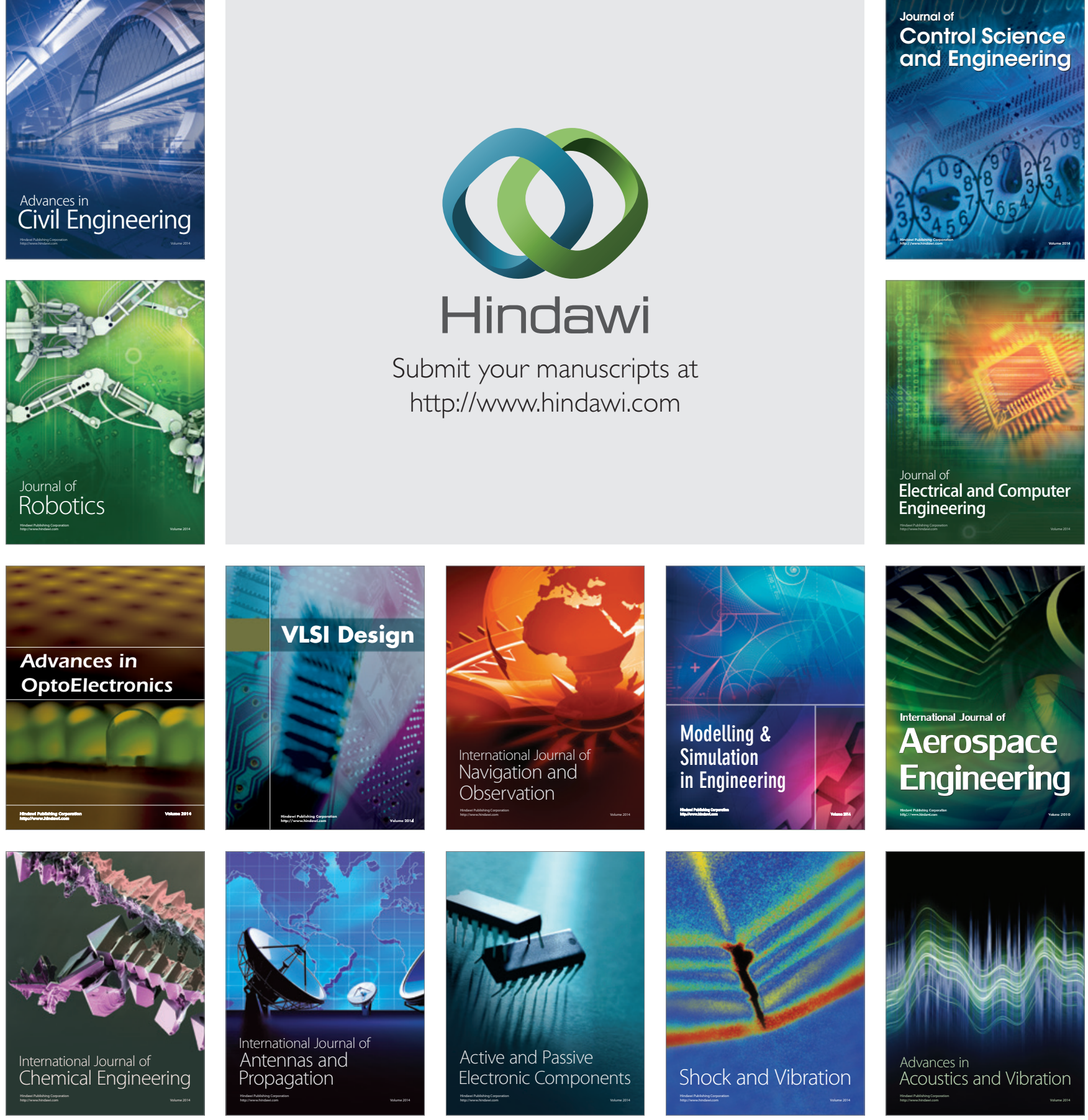